\title{
AN UNSTABLE SUPERFLUID STEWARTSON LAYER IN A DIFFERENTIALLY ROTATING NEUTRON STAR
}

\author{
C. Peralta ${ }^{1,3}$ and A. Melatos ${ }^{2}$ \\ ${ }^{1}$ Max-Planck-Institut für Gravitationsphysik, Albert-Einstein-Institut, Am Mühlenberg 1, D-14476 Golm, Germany; cperalta@aei.mpg.de \\ ${ }^{2}$ School of Physics, University of Melbourne, Parkville, VIC 3010, Australia \\ Received 2009 June 10; accepted 2009 July 17; published 2009 July 30
}

\begin{abstract}
Experimental and numerical evidence is reviewed for the existence of a Stewartson layer in spherical Couette flow at small Ekman and Rossby numbers $\left(E \lesssim 10^{-3}, R o \lesssim 10^{-2}\right)$, the relevant hydrodynamic regime in the superfluid outer core of a neutron star. Numerical simulations of a superfluid Stewartson layer are presented for the first time, showing how the layer is disrupted by nonaxisymmetric instabilities. The unstable ranges of $E$ and $R o$ are compared with estimates of these quantities in radio pulsars that exhibit glitches. It is found that glitching pulsars lie on the stable side of the instability boundary, allowing differential rotation to build up before a glitch.
\end{abstract}

Key words: dense matter - hydrodynamics - stars: interiors - stars: neutron - stars: rotation

\section{INTRODUCTION}

Meridional circulation, driven by Ekman pumping, occurs routinely in the atmospheres, oceans, and fluid interiors of rapidly rotating astrophysical objects. Indeed, it is a generic feature of Navier-Stokes flow in any spherical Couette geometry (i.e., a differentially rotating, spherical shell); see Junk \& Egbers (2000) for a review. As the Ekman number $E$ decreases and the differential rotation increases, spherical Couette flow becomes nonaxisymmetric and eventually turbulent (Nakabayashi et al. 2002). In the limit of rapid overall rotation, a detached shear layer, known as the Stewartson layer, forms along the tangent cylinder to the inner sphere (Stewartson 1957, 1966; Busse 1968). It can be disrupted by nonaxisymmetric instabilities. Numerical simulations indicate that a multiplicity of transition states are thereby excited (Hollerbach 1994, 2003; Dormy et al. 1998; Schaeffer \& Cardin 2005; Hollerbach et al. 2006).

The possible existence of an unstable Stewartson layer in a differentially rotating neutron star has important astrophysical consequences. This is true especially if the inner core rotates faster than the rest of the star, like in the Earth, a real possibility in models where the inner core makes a transition to a crystalline color-superconducting phase (Alford \& Reddy 2003; Alford et al. 2005, 2008). Recently, the importance of the global flow pattern inside a neutron star to the phenomenon of pulsar glitches has been highlighted by simulations on the vortex (Warszawski \& Melatos 2008) and hydrodynamic (Peralta et al. 2005) levels. Observational data suggest that glitches result from scale-invariant vortex avalanches driven by differential rotation (Melatos et al. 2008). If the meridional circulation is fast enough, a vortex tangle is alternately created and destroyed, producing impulsive and oscillatory torque variations (Peralta et al. 2006; Andersson et al. 2007; Melatos \& Peralta 2007). The presence of a Stewartson layer modifies these conclusions and those of other studies (e.g., of stellar oscillations), where a multicomponent superfluid is perturbed starting from a nontrivial equilibrium state (Peralta et al. 2006, 2008; Glampedakis et al. 2009; Passamonti et al. 2009).

To date, no studies have been published of the formation and stability of a Stewartson layer in superfluid spherical Couette flow. In this Letter, we present the first numerical simulation of

\footnotetext{
3 Honorary Fellow: School of Physics, University of Melbourne.
}

such a system as an idealized model of the superfluid outer core of a neutron star. We calculate stability curves for a range of unstable nonaxisymmetric modes and compare the conditions for instability with observational glitch data, finding an upper limit on the velocity shear and hence the glitch sizes observed. This Letter is organized as follows. In Section 2, we briefly review the analytic and numerical theory of Stewartson layers in viscous fluids, before calculating the structure of a steady Stewartson layer in a ${ }^{1} \mathrm{~S}_{0}$-paired neutron superfluid. In Section 3, we study the stability of the layer to nonaxisymmetric perturbations as a function of Ekman number $E$ and Rossby number $R o$. In Section 4, we compare the stability basin in the $E-R o$ plane with available glitch data. The astrophysical implications are discussed in Section 5.

\section{STEWARTSON LAYERS IN NEUTRON STARS}

Consider a viscous fluid flowing inside a differentially rotating spherical container, with inner radius (angular frequency) $R_{1}\left(\Omega_{1}\right)$, outer radius (angular frequency) $R_{2}$ $\left(\Omega_{2}\right)$, Rossby number $R o=\left(\Omega_{2}-\Omega_{1}\right) / \Omega_{2} \ll 1$, and Ekman number $E=v_{n} /\left(R_{2}-R_{1}\right)^{2} \Omega_{2}$, where $v_{n}$ denotes the kinematic viscosity. In a frame corotating with the outer sphere, the fluid outside the cylinder tangential to the inner sphere is at rest, while the fluid inside the tangent cylinder moves in a columnar fashion (Proudman 1956). Fluid is expelled (sucked in) by Ekman layers at $r=R_{2}\left(R_{1}\right)$, while a triple-deck Stewartson layer buffers the jump in angular velocity across the tangent cylinder. It consists of an inner layer of thickness $E^{1 / 3}$ sandwiched between layers of thicknesses $E^{2 / 7}\left(E^{1 / 4}\right)$ just inside (outside) the tangent cylinder (Stewartson 1966). The Ekman layers scale as $E^{1 / 2}$, except near the equator of the inner sphere, where they scale as $E^{2 / 5}$.

A superfluid Stewartson layer in a spherical shell exhibits a similar structure. Figure 1(a) graphs the angular velocity in the rotating frame as a function of the cylindrical radius $s=r \sin \theta$, for $E=1 \times 10^{-3}$ (upper curve), $1 \times 10^{-4}$ (middle curve), and $2 \times 10^{-5}$ (lower curve), with $R o=10^{-4}$. The layer starts at cylindrical radius $s \approx 1.8$ and its thickness decreases with decreasing $E$, extending out to $s \approx 2.7$ for $E=1 \times 10^{-3}$ and $s \approx 2.1$ for $E=2 \times 10^{-5}$. In viscous flows, the thickness of the layer changes by less than $1 \%$ for $0 \lesssim R o \lesssim 0.5$ (Hollerbach 2003); similar behavior is observed here. The inner Ekman layers are thicker at the equator. 


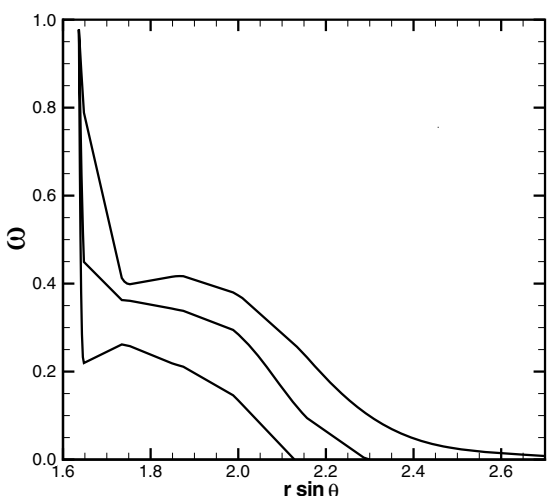

(a)

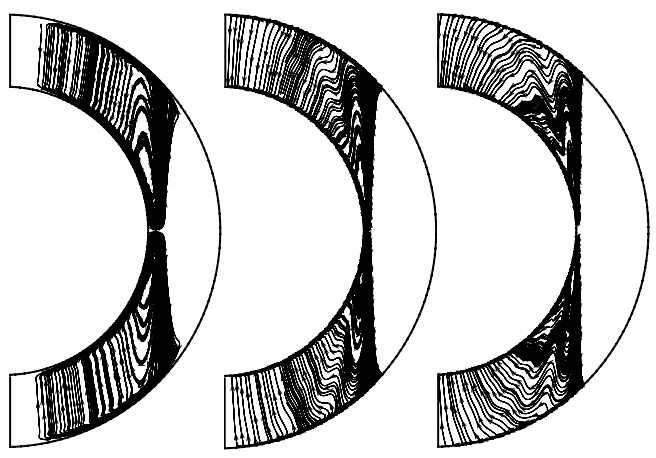

(b)

Figure 1. (a) Angular velocity $\omega$ as a function of cylindrical radius $r \sin \theta$, at $z=1.2$, for the normal fluid component in superfluid spherical Couette flow, for $E=1 \times 10^{-3}$ (upper curve), $1 \times 10^{-4}$ (middle curve), and $2 \times 10^{-5}$ (lower curve). (b) Meridional streamlines of the normal component for $E=1 \times 10^{-3}$ (left), $1 \times 10^{-4}$ (center), and $2 \times 10^{-5}$ (right). Ro equals $10^{-4}$ in all plots.

Figure 1(b) displays meridional streamlines for $E=1 \times 10^{-3}$ (left), $1 \times 10^{-4}$ (center), and $2 \times 10^{-5}$ (right), with $R o=10^{-4}$. The Stewartson layer is visible along the tangent cylinder, narrowing from left to right. The streamlines are drawn in the rotating frame of the outer sphere; the blank region to the right of the tangent cylinder indicates that the fluid there is at rest. The characteristic meridional speed in the layer scales as $E^{0.1}\left(R_{2}-R_{1}\right) \Omega_{2}$.

To obtain the results in Figure 1, we solve the two-component Hall-Vinen-Bekarevich-Khalatnikov (HVBK) equations for a superfluid inside a spherical differentially rotating shell, with $R_{1} / R_{2}=0.67$, using a pseudospectral collocation and timesplit method (Peralta et al. 2005, 2008). The details of the calculation will be set out in a longer paper. Boundary conditions assume the presence of an inner core or a transition between a ${ }^{1} S_{0}$ and ${ }^{3} P_{2}$ superfluid (Yakovlev et al. 1999; Mastrano \& Melatos 2005). We ignore vortex pinning and proton-neutron entrainment for simplicity, although recent work shows it to be important (Sedrakian \& Sedrakian 1995; Andersson \& Comer 2001). We adopt no-slip and no-penetration boundary conditions for the normal fluid component (velocity $\mathbf{v}_{n}$ ) and nopenetration for the superfluid component (velocity $\mathbf{v}_{s}$ ), ignoring the small tension force to reduce the order of the equation for $\mathbf{v}_{s}$ by one (see Henderson et al. (1995) and Peralta et al. (2008) for a discussion). The mutual friction force is taken to have the anisotropic Hall-Vinen form $\left(\propto \hat{\boldsymbol{\omega}}_{s} \times \boldsymbol{\omega}_{s} \times \mathbf{v}_{\mathrm{ns}}\right.$, with $\mathbf{v}_{\mathrm{ns}}=\mathbf{v}_{n}-\mathbf{v}_{s}$ and $\omega=\nabla \times \mathbf{v}_{s} ;$ Hall \& Vinen 1956a, 1956b), with $B=10^{-2}$, and $B^{\prime}=10^{-4}$ (Andersson et al. 2007). We take $\rho_{n} / \rho=0.01$ and $\rho_{s} / \rho=0.99$ for the normal and superfluid mass density fractions, respectively, where $\rho=\rho_{n}+\rho_{s}$ denotes the total density (Peralta et al. 2005).

\section{NONAXISYMMETRIC INSTABILITIES}

The Stewartson layer is disrupted when $R o$ exceeds a threshold $R o_{c}(E)$, which decreases as $E$ increases, exciting a KelvinHelmholtz-type instability. Hollerbach (2003) and Schaeffer \& Cardin (2005) investigated thoroughly the most unstable modes of a viscous fluid Stewartson layer. They discovered nonaxisymmetric instabilities for azimuthal modes $1 \leqslant m \leqslant 11$, with $0 \leqslant R o_{c} \leqslant 0.6$ and $10^{-10} \leqslant E \leqslant 10^{-3.5}$. The azimuthal mode number of the most unstable mode increases with decreasing $E$. Hollerbach (2003) discovered empirically the scaling $R o_{c} \sim 0.6 E^{0.65}$ for $R o>0$. For $R o<0$, the most unstable mode is almost always $m=1$, with $m=2$ being excited in the range $10^{-0.25} \lesssim R o_{c} \lesssim 0.1$. In this regime, Hollerbach (2003) found $R o_{c} \sim \widetilde{0.8 E^{0.45}}$. The asymmetry with the sign of $R o$ is still not understood physically (Hollerbach 2003).

Here, we generalize the numerical calculation of $R o_{c}$ to a superfluid Stewartson layer. We follow a two-stage recipe. First, for fixed $R o=10^{-4}$, we calculate axisymmetric HVBK basic states for $E=1 \times 10^{-3}, 1 \times 10^{-4}, 5 \times 10^{-5}, 2 \times 10^{-5}$, and $1 \times 10^{-6}$. Second, we linearize the HVBK equations around the base state and test the stability of a given $m \neq 0$ perturbation (typical amplitude $\approx 1 \%$ ) by increasing $R o$ until the mode grows exponentially. We obtain the scaling

$$
R o_{c} \approx 4.1 E^{0.40}
$$

for $10^{-6} \leqslant E \leqslant 10^{-3}$ and $0.02 \leqslant R o_{c} \leqslant 0.33$. We concentrate our efforts on $m=6$, the most unstable mode at $E \approx 10^{-3}$ for viscous fluids (and also for the superfluid). It is important to note that $m=6$ is not the most unstable mode at smaller Ekman numbers (e.g., $E=1 \times 10^{-6}$, where $m=10$ is more unstable), but the critical Rossby number is unaffected $\left(R o_{c}=0.02\right)$. In Section 3, we extrapolate the scaling Equation (1) to the regime $E \gtrsim 10^{-12}, R o \lesssim 10^{-4}$, relevant to radio pulsars (Lyne et al. 2000; Melatos \& Peralta 2007), where numerical simulations are too hard to perform. A more thorough parameter survey will be presented in a future paper.

Nonaxisymmetric instabilities can decrease the shear inside a viscous Stewartson layer by 30\% (Hollerbach et al. 2004). We observe a similar but less pronounced effect in the superfluid, e.g., when the mode $m=6$ is excited at $R o_{c}=0.3$, with $E=10^{-3.1}$. Figure 2 describes the topology of the flow in the layer, before (Figure 2(a), $R o=0.2$ ) and after (Figure 2(b), $R o=0.3)$ the mode $m=6$ is excited. The discriminant $D_{A}=$ $Q_{A}^{3}+27 R_{A}^{2} / 4$, with $R_{A}=\operatorname{det}\left(A_{i j}\right), Q_{A}=\left(A_{i j}^{2}-A_{i j} A_{j i}\right) / 2$, and $A_{i j}=\partial v_{i} / \partial x_{j}$, distinguishes between regions that are focal $\left(D_{A}>0\right.$, blue) and strain-dominated $\left(D_{A}<0\right.$, green) (Chong et al. 1990). The maximum shear changes from $d \omega / d s=1.1$ at $t=0$ to $d \omega / d s=1.0$ at $t=400$ (compared to viscous fluid, where the observed change is from $d \omega / d s=1.3$ to 0.8 ). The hexagonal flow structure also boosts the torque one must exert on the inner sphere to maintain the shear, although the increase is less dramatic $(\sim 10 \%)$.

\section{PULSAR GLITCHES}

As $E$ and Ro control the stability of the Stewartson layer, it is interesting to test whether the amplitude and rate of incidence 
(a)

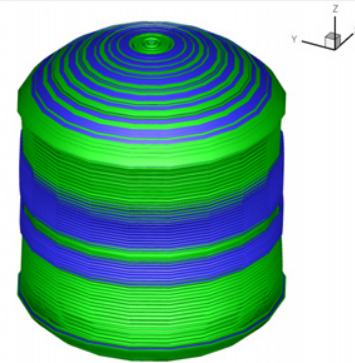

(b)

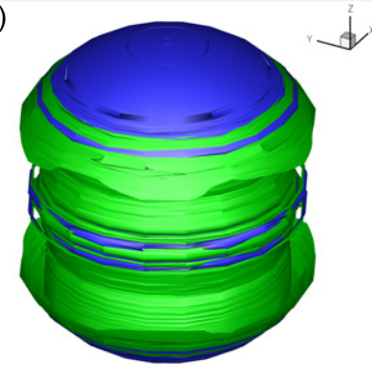

Figure 2. Isosurfaces of the velocity gradient discriminant (see the text): $D_{A}=10^{-3} \Omega_{2}^{6}$ (blue) and $-10^{-3} \Omega_{2}^{6}$ (green) for $E=10^{-3.1}$ and the transition $R o=0.2 \rightarrow 0.3$, where $m=6$ is the most unstable mode: (a) $t=0$ (before transition); (b) $t=400$ (after transition).

of rotational irregularities like glitches are related to these two dimensionless quantities. In order to calculate $E$, we need to know how $v_{n}$ depends on the density $\rho$ and temperature $T$ in the outer core. Using the neutron-neutron scattering viscosity formula derived by Cutler \& Lindblom (1987), we find

$$
E=2.6 \times 10^{-12}\left(\frac{T}{10^{8} \mathrm{~K}}\right)^{-2}\left(\frac{\Omega_{2}}{10^{2} \mathrm{rad} \mathrm{s}^{-1}}\right)^{-1}
$$

with $\rho=2.8 \times 10^{12} \mathrm{~g} \mathrm{~cm}^{-3}$. The core temperature $T$ is related to the surface temperature $T_{s}$, e.g., via the two-zone heat-blanket model of Gudmundsson et al. (1982), which gives $T / 10^{8} \mathrm{~K}=$ $1.29\left(T_{s} / 10^{6} \mathrm{~K}\right)^{1.8}$. We estimate $T_{s}$ from the characteristic age $\tau_{c}=\Omega_{2} / 2\left|\dot{\Omega}_{2}\right|$, combined with theoretical cooling curves for $\tau_{c} \leqslant 10^{6}$ yr (Page 1998) and standard neutrino cooling; similar $E$ distributions are obtained with nonstandard cooling (Melatos $\&$ Peralta 2007).

In Figure 3, we plot as points the maximum $R o$ in 55 glitching pulsars with $\tau_{c} \leqslant 10^{6} \mathrm{yr}$, taken from Table 1 in Melatos et al. (2008), assuming conservatively that $R o$ is less than the fractional frequency jump of the largest glitch. For each object, $E$ is estimated by the method in the previous paragraph. We also plot two $R o_{c}(E)$ curves: the HVBK scaling computed in Section $2\left(R o_{c} \approx 4.1 E^{0.40}\right.$, solid curve $)$ and a scaling extrapolated from the study by Schaeffer \& Cardin (2005) for $R o_{c} \lesssim 10^{-2}$ and $E \lesssim 10^{-5}\left(R o_{c} \approx 9.4 E^{0.57}\right.$, dashed curve). The viscous fluid scaling lies in the middle of the cluster of points, while the HVBK superfluid scaling lies above all the points. This suggests two possible conclusions: (1) a glitching pulsar must have $R o<R o_{c}(E)$, otherwise Stewartson layer instabilities would erase the shear required for the glitch phenomenon to occur and (2) it is important to include the HVBK superfluid dynamics to ensure that all points in Figure 3 lie below the $R o_{c}(E)$ curve, given that no discernible difference is observed in the glitch behavior of objects above and below the viscous fluid scaling.

In the superfluid Stewartson layer, there is an extra ingredient that influences the instability curve: the mutual friction between the normal and superfluid component, controlled by the dimensionless parameters $B$ and $B^{\prime}$. We have only considered the weak coupling regime in this investigation $\left(B=10^{-2}\right.$, $\left.B^{\prime}=10^{-4}\right)$. In the strong coupling regime $\left(B=0, B^{\prime}=1\right.$; Andersson et al. 2007), the instabilities are likely to be quite different. Preliminary results for axisymmetric steady states show that the Stewartson layer is $\sim 10 \%$ thicker. A more detailed investigation of the effect of mutual friction and entrainment on $R o_{c}(E)$ will be presented in a future paper.

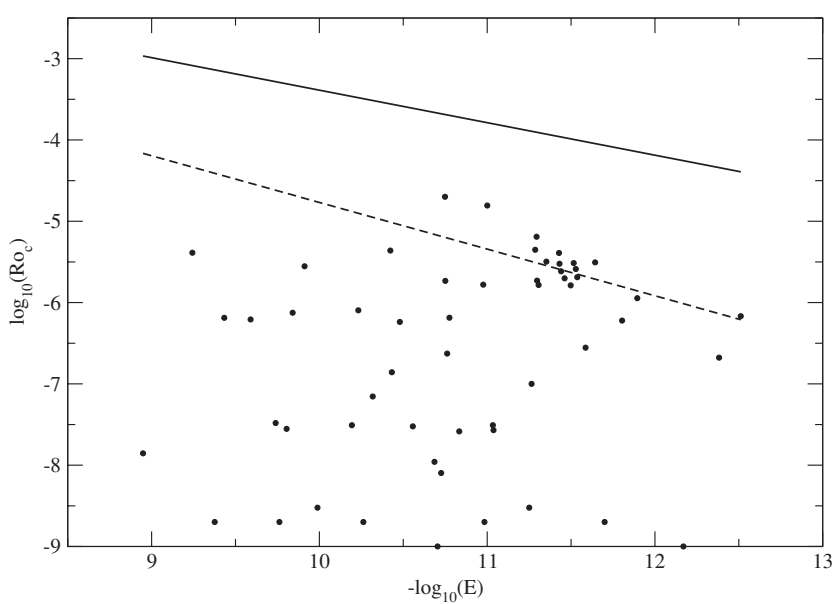

Figure 3. Critical Rossby number $R o_{c}$ vs. Ekman number $E$ for 55 glitchers with characteristic ages $\tau_{c} \leqslant 10^{6} \mathrm{yr}$. The points indicate upper limits on $R o_{c}$ derived from the largest observed glitch in each object. $E$ is calculated using standard neutrino cooling. The solid curve is a fit to the HVBK superfluid simulations in Sections 2 and 3. The dashed curve is an extrapolated fit to the viscous fluid calculations from Schaeffer \& Cardin (2005).

\section{DISCUSSION}

The results in Sections 2 and 3 demonstrate that Stewartson layers develop in rotating HVBK superfluids, like the ${ }^{1} S_{0}$-paired neutron superfluid in the outer core of a neutron star. We present the first numerical simulation of a HVBK Stewartson layer, for Ekman and Rossby numbers in the ranges $10^{-6} \leqslant E \leqslant 10^{-3}$ and $10^{-4} \leqslant R o \leqslant 10^{-2}$. Superfluid Stewartson layers are unstable to nonaxisymmetric perturbations. Transitions between unstable Stewartson modes in the superfluid are different to those found in a viscous fluid, and the critical Rossby number $R o_{c}(E)$ is $\sim 10$ times higher in the above parameter regime. In glitching pulsars, one finds $R o<R o_{c}(E)$ in the 55 pulsars for which $R o$ and $E$ can be estimated reliably from observations. One possible interpretation of these data is that the Stewartson layer remains stable in these objects, allowing rotational shear to build up (as required for glitches) without triggering disruption of the Stewartson layer (which would nullify the shear (Hollerbach et al. 2004) and hence shut down the glitching behavior). The threshold $R o_{c}(E)$ can be compared against the upper limit derived independently from the gravitational-wave spin down caused by Kolmogorov-like superfluid turbulence excited in the stellar interior (Melatos \& Peralta 2009).

The conclusions drawn from the data in Figure 3 extend and partially clarify the surprising empirical finding, that the $E$ distribution is markedly different between glitchers and nonglitchers (Melatos \& Peralta 2007). It seems strange that stars with $E \sim 10^{-10}$ (few glitches) and $E \sim 10^{-12}$ (many glitches) behave so differently, since in both regimes Kolmogorov turbulence must be fully developed and scale-free. However, from Figure 3 (and Figure 4 in Hollerbach 2003), one sees that small differences in $E$ and $R o$ lead to very different flow states and stability properties (Hollerbach 2003; Hollerbach et al. 2004). Moreover, if theoretical estimates of effective viscosity and hence $E$ are too low by a factor of $\sim 10^{5}$, due to turbulent Reynolds stresses (Melatos \& Peralta 2007), glitching pulsars lie in a range where (1) the most interesting flow transitions occur before the flow becomes turbulent (i.e., at $10^{-4} \lesssim E \lesssim 10^{-2}$ for $1 \leqslant m \leqslant 6$ ) and (2) numerical simulations are computationally tractable. 
The results of this Letter do not prove that Stewartson flow transitions control glitch behavior. We merely find empirically that all observed glitchers lie on the stable side of the $R o_{c}(E)$ threshold for nonaxisymmetric instabilities of a Stewartson layer in an HVBK superfluid. Recently, a hydrodynamic trigger for pulsar glitches was proposed by Glampedakis \& Andersson (2009), associated with $r$-modes excited by differential rotation. It would be interesting to see how meridionally circulating Stewartson base states modify these calculations, especially in the strong pinning scenario (for which $B^{\prime}=1, B=0$ ). Price et al. (2008) found empirical evidence of departures from solidbody rotation in a radio pulsar.

We do not consider stratification or magnetic fields in this investigation. In viscous fluids, strong stratification suppresses the Stewartson layer, leaving a Taylor column parallel to the rotation axis (Hollerbach 2009). Magnetic fields widen the Stewartson layer, merging it with the interior flow (Hollerbach 1994). This difficult physics deserves investigation.

We acknowledge the support of the Max-Planck Society (Albert-Einstein Institut) and the computer time supplied by the Victorian Partnership for Advanced Computation. We thank Rainer Hollerbach for indicating the importance of the Stewartson layer and very illuminating discussions. This work made use of the ATNF Pulsar Catalogue (http://www.atnf.csiro.au/research/pulsar/psrcat; Manchester et al. 2005).

\section{REFERENCES}

Alford, M., Jotwani, P., Kouvaris, C., Kundu, J., \& Rajagopal, K. 2005, Phys. Rev. D, 71, 114011

Alford, M., \& Reddy, S. 2003, Phys. Rev. D, 67, 074024

Alford, M. G., Schmitt, A., Rajagopal, K., \& Schäfer, T. 2008, Rev. Mod. Phys., 80,1455

Andersson, N., \& Comer, G. L. 2001, Phys. Rev. Lett., 87, 241101

Andersson, N., Sidery, T., \& Comer, G. L. 2007, MNRAS, 381, 747

Busse, F. H. 1968, J. Fluid Mech., 33, 577
Chong, M. S., Perry, A. E., \& Cantwell, B. J. 1990, Phys. Fluids, 2, 765 Cutler, C., \& Lindblom, L. 1987, ApJ, 314, 234

Dormy, E., Cardin, P., \& Jault, D. 1998, Earth Planet. Sci. Lett., 160, 15

Glampedakis, K., \& Andersson, N. 2009, Phys. Rev. Lett., 102, 141101

Glampedakis, K., Andersson, N., \& Jones, D. I. 2009, MNRAS, 359, 1908

Gudmundsson, E. H., Pethick, C. J., \& Epstein, R. I. 1982, ApJ, 259, L19

Hall, H. E., \& Vinen, W. F. 1956a, Proc. R. Soc. Lond. A, 238, 204

Hall, H. E., \& Vinen, W. F. 1956b, Proc. R. Soc. Lond. A, 238, 215

Henderson, K. L., Barenghi, C. F., \& Jones, C. A. 1995, J. Fluid Mech., 283, 329

Hollerbach, R. 1994, Proc. R. Soc. Lond. A, 444, 333

Hollerbach, R. 2003, J. Fluid Mech., 492, 289

Hollerbach, R. 2009, Phys. Lett. A, submitted

Hollerbach, R., Futterer, B., More, T., \& Egbers, C. 2004, Theor. Comput. Fluid Dyn., 18, 197

Hollerbach, R., Junk, M., \& Egbers, C. 2006, Fluid Dyn. Res., 38, 257

Junk, M., \& Egbers, C. 2000, in Lecture Notes in Physics 549, Physics of Rotating Fluids, ed. C. Egbers \& G. Pfister (Berlin: Springer), 215

Lyne, A. G., Shemar, S. L., \& Smith, F. G. 2000, MNRAS, 315, 534

Manchester, R. N., Hobbs, G. B., Teoh, A., \& Hobbs, M. 2005, Astron. J., 129, 1993

Mastrano, A., \& Melatos, A. 2005, MNRAS, 361, 927

Melatos, A., \& Peralta, C. 2007, ApJ, 662, L99

Melatos, A., \& Peralta, C. 2009, ApJ, submitted

Melatos, A., Peralta, C., \& Wyithe, J. S. B. 2008, ApJ, 672, 1103

Nakabayashi, K., Tsuchida, Y., \& Zheng, Z. 2002, Phys. Fluids, 14, 3963

Page, D. 1998, in Frontiers Science Ser. 24, Neutron Stars and Pulsars: Thirty Years after the Discovery, Proc. International Conf. on Neutron Stars and Pulsars, ed. N. Shibazaki et al. (Tokyo: Universal Academy Press), 183

Passamonti, A., Haskell, B., Andersson, N., Jones, D. I., \& Hawke, I. 2009, MNRAS, 394, 730

Peralta, C., Melatos, A., Giacobello, M., \& Ooi, A. 2005, ApJ, 635, 1224

Peralta, C., Melatos, A., Giacobello, M., \& Ooi, A. 2006, ApJ, 651, 1079

Peralta, C., Melatos, A., Giacobello, M., \& Ooi, A. 2008, J. Fluid Mech., 609, 221

Price, S., Link, B., \& Shore, S. 2008, APS Meeting Abstracts, B5008

Proudman, I. 1956, J. Fluid Mech., 1, 505

Schaeffer, N., \& Cardin, P. 2005, Phys. Fluids, 17, 104111

Sedrakian, A. D., \& Sedrakian, D. M. 1995, ApJ, 447, 305

Stewartson, K. 1957, J. Fluid Mech., 3, 17

Stewartson, K. 1966, J. Fluid Mech., 26, 131

Warszawski, L., \& Melatos, A. 2008, MNRAS, 390, 175

Yakovlev, D. G., Levenfish, K. P., \& Shibanov, Y. A. 1999, Usp. Fiz. Nauk, 42, 737 